\title{
COMPRESIÓN MEDULAR DORSAL EXTRADURAL POR CONDKOMA
}

\author{
B. B. Srota \\ C. A. BARDECI \\ J. C. ChristenseN
}

Las neoformaciones medulares son de observación poco frecuente alcanzando su incidencia, en forma aproximada, a solo el $2 \%$ del total de lesiones neurológicas. A su vez los distintos tipos histológicos de tumores que se encuentran en la práctica, muestran un porcentaje de frecuencia ell extremo diferente.

Esta afirmación ha sido ampliamente demostrada por Elsberg ${ }^{1}$, quien, en una estadística sobre sobre 275 casos de tumores medulares, asigna a los intradurales extramedulares un $65 \%$ de frecuencia, a los extradurales un $20 \%$ y a los intramedulares solo un $15 \%$. Dentro de los tumores extradurales se encuentran los condromas, de observación en extremo rara y que pueden localizarse en la cavidad craneana a punto de partida meníngeo (meningiomas condroblásticos de Cushing) o asentar en el trayecto vertebral; a estos últimos nos referiremos en este trabajo.

La bibliografía de estos blastomas es escasa, en particular la del condroma vertebral. Foderl en $1897^{2}$ se refiere por primera vez a un condroma de vértebra que asentaba en los tejidos paravertebrales sin sintomatología medular. La primera publicación de un condroma de vértebra con compresión medular corresponde a Bruno en $1913^{3}$. Hannemann se refiere ${ }^{4}$, en 1919, a un condrosarcoma del atlas con compresión aguda de la médula cervical. May ${ }^{5}$ publica en 1927 un condroma de vértebra con signos compresivos, mientras Desjacques ${ }^{6}$ refiere otro en los tejidos paravertebrales sin síntomas neurológicos. Elsberg, en 1928, sobre 179 tumores incluye 11 condromas sin especificar su tipo. Paulian y Bistriceano ${ }^{7}$ presentan a la Sociedad de Neurología de Paris, en 1936, un caso de condroma osificante con paraplejia espástica. En cuanto a los condromas del disco intervertebral, luego de una breve referencia de Adson ${ }^{8}$, en 1925, son estudiados por Stookey ${ }^{9}$, en 1928 , quien establece su sintomatología en un documentado trabajo con 7 casos, todos a localización cervical. Le siguen Von Peky ${ }^{10}$, Alajouanine y Petit-Dutaillis ${ }^{11}$ y Bucy ${ }^{12}$, quien recopila hasta 1930 , entre casos publicados y referidos, 16 condromas del disco intervertebral, algunos de los cuales asentaban también en la región dorsal y lumbar.

Trabajo del Servicio de Clínica Neurológica del Hospital Español (Jefę: Prof. Dr. B. B. Spota), comunicado a la Socicdad de Neurologia, Psiquiatria y Neurocirugia de Buenos Aires en el 19 de agosto de 1949. 
Nuestro caso, un condroma de vértebra, une a su rara frecuencia algunas características clínicas poco comunes que, junto al éxito quirúrgico con la recuperación funcional completa del paciente, hacen interesante su consideración.

Q. M., español, de 51 años de edad, casado, agricultor. Ingresa al Servicio el 26 de abril de 1949. Enfermedad actual: Comienza en el mes cle octubre de 1948, en pleno estado de salud; a raí\% de un pequeño accidente ocurrido a una de sus hijas sale corriendo hacia ella y a los 30 ó 40 metros se detiene bruscamente por claudicación motriz de sus miembros inferiores que dura aproximadamente $10 \mathrm{minu}$ tos, al cabo de los cuales puede reanudar la marcha en torma normal. Después de este episodio queda libre de síntomas durante unos días. A continuación aparecen progresivas parestesias en forma de hormigueos y sensación de enfriamiento en ambos pies que se propagan hacia las piernas y muslos. Continúa con estos síntomas y a los tres meses se agrega ligera impotencia motriz en los miembros inferiores que le dificultaban la marcha. Esta impotencia se fué acentuando y a los dos meses de su comienzo la ambulación se hizo imposible. Quince días antes de su ingreso podía permanecer de pie, aunque con gran dificultad y desde su internación la impotencia fué absoluta. Antes de ingresar a nuestro Servicio estuvo internado en un sanatorio particular donde le practicaron punción lombar y exámenes que citaremos en el estado actual. Antecedentes hereditarios sin importancia. Antecedentes personales: Sarampión a los 21 años. Blenorragia a los 26 años. Casó a los 39 años con esposa aparentemente sana. Un hi,jo falleció al nacer en parto prematuro de 8 meses. Otro falleció a los 7 días, ignora la causa. Uno vive y es sano. Colecistitis y congestión pulmonar a los 46 años. Fumador de 30 cigarrillos diarios. Bebedor de medio litro de vino por día.

Examen neurológico: Constitución pícnica acentuada. Obeso. Trofismo normal. Abdomen prominente, en obús, más acentuado en su parte inferior. Hipertonía marcada piramido-extrapiramidal en ambos miembros inferiores a predominio en el lado izquierdo. Motilidad pasiva: aumento de la excursión del lado izquierdo (Lesión cordonal posterior superpuesta). Motilidad activa: súlo ligero movimiento de flexión del muslo sin mantener la extensión de la pierna. La fuerza está abolida en ambos miembros inferiores. Los reflejos osteotendinosos están conservados en los miembros superiores. En los inferiores: hiperreflexia, clonus y Babinski bilateral a predominio izquierdo. Reflejos de automatismo medular en ambos lados. Intensas perturbaciones de la sensibilidad propioceptiva en ambos miembros inferiores, más intensa del lado izquierdo. Hipoestesia e hipoalgesia bilateral con nivel superior en la $V$ metámera dorsal y más acentuadas del lado derecho (Brown-Sequard izquierdo).

Punción lumbar: (realizada antes de su ingreso). Maniobra de Queekenstedt: bloqueo parcial. Examen de L.C.R.: Albumúmina 0,78 grs. \% ${ }^{\circ}$ Glucosa 0,53 grs. $\%{ }_{00}^{\circ}$ Elementos $1,6 \mathrm{~mm}^{3}$. Wasserman negativa. Guillain 0000222222222200.

Con estos elementos clínicos y de laboratorio que configuraban un síndrome de compresión medular dorsal, solicitamos una radiografía de columna cuya imagen mostró (fig. 1) la existencia de una sombra tumoral, redondeada, irregular, a la altura de la VI vértebra dorsal, con calcificaciones de aspecto grumoso, lateralizada hacia la izquierda, con osteolisis de casi toda la hemivértebra izquierda, desaparición de la sombra de implantación del pedículo de ese lado, estando los discos respetados. Además, a distintas alturas, formaciones osteofíticas, algunas de tipo pico de loro y otras como la de la VIII dorsal del lado izquierdo, de características inusitadas de verdadero mamelón óseo y otras neoformaciones de costillas que pudimos interpretar a posteriori.

Con presunción diagnóstica de un probable angioma, por desconocimiento de la imagen radiográfica típica, se resolvió la intervención, que por falta de material fué realizada con los datos clínicos y radiográficos mencionados. 


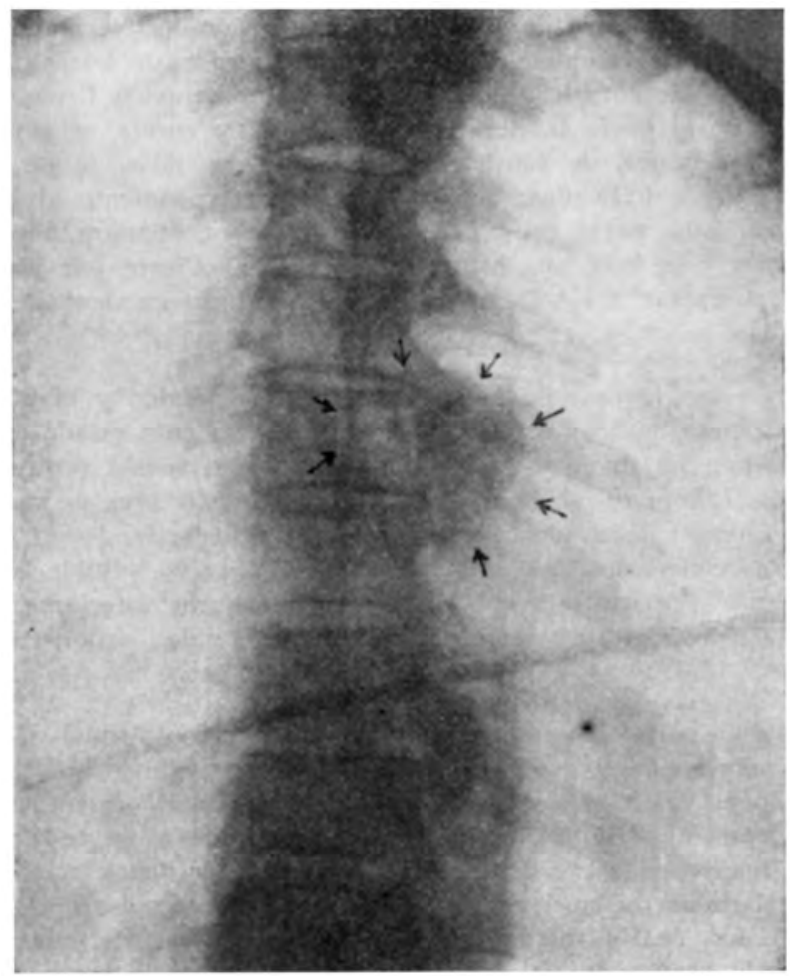

Fig. 1 - Caso Q. M. Radiografia mostrando sombra tumoral en la altura de la VI vertebra dorsal.

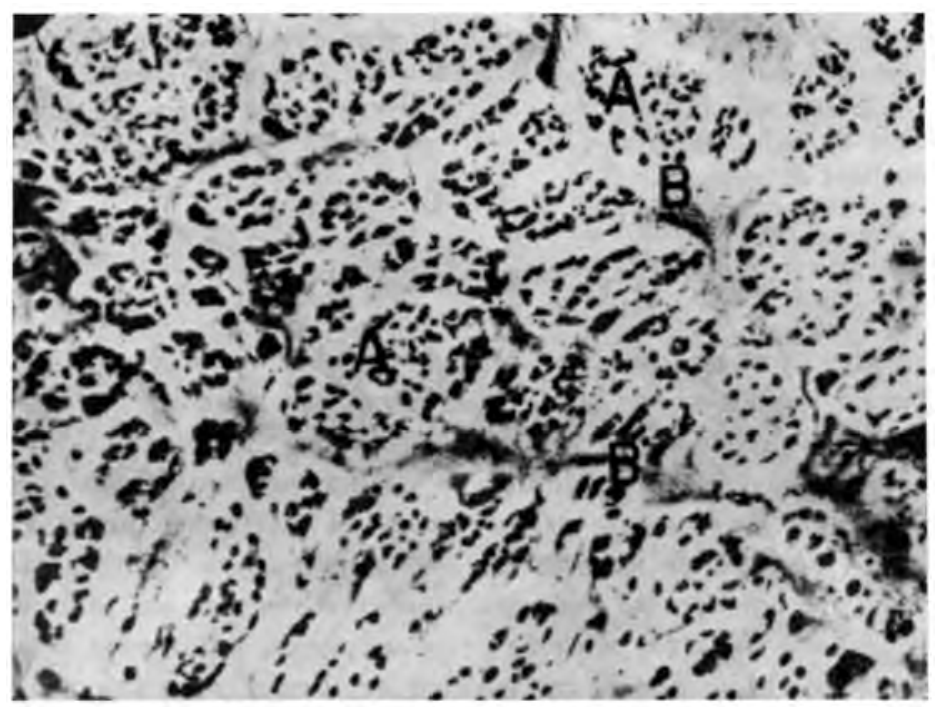

Fig. 2 - Caso Q. M. Microfotografia del material extirpado cirugicamente. Condroma hialino (descripcion en el texto). 
Operación: El 9 de Mayo de 1949. Anestesia general suplementada con anestesia local. Laminectomía completa de 5.* y 6.* dorsales y de bordes adyacentes de la 4." y 7.*. A nivel de dorsal 6." se encuentra una tumoración firme, izquiercla, que desplaza el saco dural hacia la derecha. Con gubia y cureta se extirpa el tumor completamente. El tumor, de consistencia firme y granulosa, es de aspecto cartilaginoso y con zonas calcificadas; ocupa la zona correspondiente al pedículo vertebral e invade asimismo parte del cuerpo de la vértebra. Sangra poco. Se curetea el lecho del tumor y se hace una hemostasia perfecta. Cierre por planos. Drenaje extradural que se extrae a las 24 horas. Post-operatorio excelente. Los puntos se sacan a los 7 días.

Evolución: A los 10 días de operado el enfermo camina y el 27 de junio, un mes y 18 días después de la operación, se levanta el siguiente estado actual: Camina 40 a 50 cuadras por día sin fatigarse. La motilidad activa está recuperada en todos los movimientos. Ligera disminución de fuerzas en los flexores de pierna izquierda. En la derecha fuerza conservada. Persiste la hiperreflexia tendinosa más acentuada del lado izquierdo con clonus, que se agota del lado derecho. Babinski bilateral más del lado izquierdo. Hiperestesia cutánea en los miembros inferiores. Ligera hipokinestesia a izquierda. Estatestesia conservada. Sensibilidad protopática y epicrítica conservadas.

Examen auátomopatologico: (Dr. Polak) Fijación en formol al 10\%. Cortes obtenidos por congelación y por previa inclusión en parafina. Coloración: azul de anilina de Mallory; tricrómico de Masson e impregnaciónes argéntica de Río Hortega. Histológicamente (fig. 2) el material corresponde a un tejido neoformativo de estructura condromatosa, constituído por amplios cordones celulares separados entre sí por trabéculas conjuntivas surcadas por vasos sanguíneos. Las células cartilaginosas $(\Lambda)$, son de tipo hialino y se disponen con relativa regularidad aisladamente o formando grupos de 2 y 4 elementos. La substancia fundamental (B) que rodea a las células es de tipo mucoide o hialino, en zonas ligeramente basófila y en otras con incipiente acidofilia. Histopatológicamente se diagnostica Condroma Hialino.

Síntesis clínica: Compresión medular dorsal extradural a la altura de la VI vértebra, a comienzo de claudicación intermitente medular de Dejerine, de curso indoloro y evolución en 6 meses desde el comienzo de los síntomas hasta la paraplejía completa. Exéresis quirúrgica completa con recuperación funcional prácticamente total. Tumor de tipo condroma vertebral con invación del canal raquídeo y signos de condromatosis de columna.

\section{COMENTÁRIO}

El comienzo por un episodio típico de claudicación intermitente medular de Dejerine constituye una de las características más salientes de la sintomatología de nuestro enfermo a la par que un síntoma extraordinariamente raro en la clínica de los tumores medulares. Algunas neoformaciones, come ciertos fibroblastomas, sarcomas y especialmente el carcinoma metastásico, pueden presentar, después de un esfuerzo o de una punción lumbar, una paraplejía brusca no transitoria, casi siempre precedida por un periodo más o menos prolongado de otros síntomas como dolores, parestesias y trastornos vegetativos. Se adnite que la malignidad del tumor y su asiento en el es- 
pacio extradural, al ocasionar una interferencia en el aflujo sanguíneo a la médula con su consiguiente mielomalacia, serían las causas determinantes de dicha paraplejía.

La claudicación intermitente medular descripta por el famoso neurólogo francés y de observación frecuente en las afecciones medulares inflamatorias - meningomielitis específica, esclerosis en placas, etc. - se atribuye a la isquemia medular que ocasiona la lesión meningovascular de dichos procesos al impedir la vasodilatación arterial necesaria cuando el órgano aumenta su actividad.

Un mecanismo patogénico similar nos permite explicar la claudicación de nuestro enfermo: el crecimiento asintomático del tumor en el espacio extradural fué limitando las posibilidades dinámicas de ađaptación del sistema circulatorio perimedular y al realizar el enfermo un esfuerzo violento, se produjo la isquemia medular transitoria que configuró una típica claudicación intermitente.

La ausencia de dolores en toda la evolución de la enfermedad de nuestro paciente constituye otro hecho de rara observación. El dolor aparece como primer síntoma en el 80 a $90 \%$ de los tumores medulares. Su ausencia es excepcional sobre todo en los intradurales extramedulares (Almeida Lima $\left.{ }^{13}\right)$. Elsberg, sobre 100 casos encontró 8 sin dolor, de los cuales 3 eran intramedulares y 5 extramedulares. En los extradurales el dolor radicular precoz es menos frecuente que en los intradurales porque en éstos las relaciones del tumor con las vainas o raíces nerviosas es mucho más estrecha que en aquellos.

Además en los extradurales la interposición de la dura y del I.C.R. entre el tumor y las estructuras radículo-medulares hacen posible la existencia de formas indoloras, por lo menos al comienzo. Estas circunstancias, la posición lateral del tumor, en nuestro caso, y el papel que asignamos a los trastornos circulatorios en la génesis de la sintomatología, permiten explicar la falta de dolores.

Con respecto ao nivel de los trastornos sensitivos en relación con la altura del tumor, encontramos un hecho sobre el cual Elsberg llanó la atención: de que en ciertos tumores extradurales y en forma inconstante, existía una evidente disociación siringomiélica y mayor intensidad lesional en los segmentos proximales al tumor. La localización extramedular de estos tumores, su posición dorsolateral frecuente y la compresión de la médula de afuera hacia adentro, determinan mayores lesiones en las fibras sensitivas de las metámeras distales, que se disponen en la parte póstero-externa del haz espino-talámico, de ahí que habitualmente el nivel de los trastornos sensitivos suele ser inferior al de localización del tumor. La congruencia de ambos niveles ha sido explicada en virtud de la sinapsis de las fibras de la sensibilidad térmico dolorosa y táctil protopática con las neu- 
ronas del asta posterior, atribuyéndose a la substancia gris de éstas una menor resistencia cuando se ejerce una compresión global sobre la médula.

Las dificultades diagnósticas que pudo plantear la evolución indolora en nuestro enfermo, fueron superadas por la nanometría lumbar y la disociación albúmino-cilológica en el L.C.R. que afirmaron el diagnóstico de compresión medular. Cuando ésta se debe a un tumor extradural es frecuente, según Elsberg, que se agraven los síntomas después de la raquicentesis.

Por otra parte, la radiología suele ser de gran utilidad para el diagnós. tico de los tumores extradurales por la frecuencia de los cambios óseos, el ensanchamiento del espacio interpedicular o la existencia de calcificaciones. En lo que al condroma se refiere, la imagen es típica y permite su diagnóstico diferencial; es de forma redondeada, densa, de aspecto grumoso, en la que se destacan sombras de mayor densidad, irregulares, pequeñas, que la distinguem de la imagen del hemangioma vertebral de Bailey y Bucy con calcificaciones a disposición radiada a partir de un núcleo común y con trabeculación ósea grosera. En los condromas de vértebra no suelen faltar los signos de destrucción ósea. Por otra parte, el hemangioma muy raramente dá signos de compresión medular. El carcinoma metastásico es siem. pre doloroso, de evolución rápida y los antecedentes así como el examen radiográfico permiten identificarlo.

Los condromas pueden clasificarse, siguiendo a Elsherg, de acuerdo con su localización, en extradurales primitivos o secundarios. Los primeros se localizan dentro del canal raquídeo desde el comienzo, mientras los últimos lo invaden secundariamente.

Los primitivos pueden tomar su origen en las meninges o en el disco intervertebral. Los meningeos han sido designados por Cushing como meningiomas condroblásticos, suelen ser intracraneanos, habiendo descripto Flsberg uno espinal, adherido al lado ventral de la dura, sin conexión con el disco o el hueso. Los condromas del disco intervertebral fueron estudiados por Stookey, quien describió 7 casos, todos localizados en la región cervical. Se hallan sobre el disco fijos en la pared anterior del canal. Generalmente pequeños, de 1 a $11 / 2$ cms. dan síntomas de compresión medular anterior, atrofia de uno o dos segmentos cervicales a nivel del tumor con espasticidad homolateral y trastornos de la sensibilidad térmico-dolorosa heterolateral, sin alteraciones propioceptivas. Publicaciones posteriores (Bucy, Yon Pecky) describen condromas del disco a localización dorsal y lumbar.

Los condromas extradurales secundarios asientan en el cuerpo vertebral desde el cual invaden el canal raquídeo generalmente por destrucción ósea. Nuestro caso mostró en la intervención la fuerte adherencia del tumor al pedículo de la VI vértebra dorsal invadiendo hacia adentro el canal, donde por compresión directa e interferencia en el aporte sanguíneo dió lugar a la interrupción funcional de los haces medulares. 
Por otra parte el condroma invadió los tejidos paravertebrales como se aprecia en la radiografía. Foderl y Desjacques han descripto sendos condromas de vértebra que asentaban exclusivamente en dichos tejidos. En cuanto al origen de estos tumores se adnite que resultan de la proliferación metaplásica del tejido osteoformador ya sea de la médula ósea o del periostio. El examen anátomopatológico muestra la existencia de acúmulos de cartílago con verdaderos lóbulos de células condroblásticas separadas por espacios intercelulares de constitución diversa; en nuestro enfermo se apre. cia una substancia intercelular homogénea de tipo hialino. Puede hallarse una forma fibrosa o bien elástica en las que la substancia fundamental se halla atravesada por fascículos conjuntivos o fibras elásticas respectivamente, que atraviesan los grupos de células cartilaginosas. A la inversa del tejido cartilaginoso normal, los condromas son vascularizados, como puede observarse en la microfotografía de la figura $n .^{\circ} 3$ de nuestro caso. Es muy frecuente el depósito de sales calcáreas cuya disposición da la imagen radiológica característica.

La evolucion es más rápida en los condromas de vértebra (alrededor de los 6 meses) que en los de disco, de ahí la importancia del diagnóstico precoz - antes que se produzcan lesiones medulares compresivas irreversibles - para el éxito quirúrgico. El pronóstico, dada la constitución histológica de estos tumores. es bueno. El resultado de la intervención y la evolución ulterior inmediata de nuestro paciente, han sido excelentes. La existencia de otras neoformaciones de aspecto condroblástico en otras vértebras y en algunas costillas, asintomáticas en el momento actual, plantean un interrogante con respecto al pronóstico alejado de nuestro caso.

\section{CONCLUSIONES}

Se describe por primera vez en la bibliografía nacional, un condroma de vértebra con compresión medular dorsal extradural. Se destacan las características clínicas de comienzo por una claudicación intermitente medular de Dejerine y la evolución indolora hasta la intervención quirúrgica. Se pone al día la bibliografía y se estudian los signos radiográficos y la anatomía patológica de los condromas.

SUMMARY

For the first time in Argentine bibliography a chondroma of the vertebra with extradural dorsal medular compression, is described. The clinical characteristics of the onset by intermitent medular claudication of Dejerine and the painless evolution until surgical intervention, are stressed. The bibliography is put up to date and the radiographics signs and the pathologic anatomy of the chondromas, are studied. 


\section{BIBLIOGRAFIA}

1. Elsberg, C. A. - a) Extradural spinal tumors (primary, secondary, ametastatic). Surg., Gynec, a. Obst., 46:1, 1928, b) Tumors of the spinal cord. Problems in their diagnosis and localization; procedures for their exposure and removal. Arch. Neurol. a. Psychiat., 22:949, 1929. c) Surgical diseases of the spinal cord. Paul B. Hoeber, Inc., New York, 1941.

2. Foderl, O. - Enchondrom der Halswirbelsäule. Deutsch. Ztschr. f. Chir., 45: 154-159, 1897.

3. Bruno, V. - Enchondrom der wirbelsäule. Beitr. z. Klin. Chir., 85:124-130, 1913.

4. Hannemann, E. - PIötzlicher Tod infolje Kompression des obersten Halsmarkes durch ein Chondrosarkom des Atlas. Deutsch. 'Ttschr. f. Nervenk, 63:251-256, 1919.

5. May, R. J. - Chondroma of vertebra. Report of case. Am. J. Roentgenol., $17: 452-455,1927$.

6. Desjacques, R. -.- A propos d'un cas de chondrome de la colomne cervicale. Lyon Chir., 24:40-43, 1927.

7. Paulian, D. e Bistriceano, I. - Chondrome ossifiant extraduremérien du rachis dorsal inferieur, avec paraplegie spastique consecutive. Etude anatomo-clinique. Rev. Neurol., 65:989-997, 1936.

8. Adson, A. W. - Diagnosis and treatment of tumors of the spinal cord. Northwest Med., 24:309-317, 1925.

9. Stookey, 13. - Compresion of the spinal cord due to ventral extradural cervical chondroma. Arch. Neurol. a. Psychiat., 20:275, 1928.

10. Von Pecky, K. - Zur Kenntnis der gutartigen wirbelsäulenje-schwülste in Wirbelkanal. Frankfurt '/tschr. f. Path., 37:562-567, 1929.

11. Alajouanine e Petit Dutaillis - Bull. et Mem. Soc. Nat. de Chir., 55:937-945, 1929 .

12. Bucy, P. C. -- Chondroma of intervertebral disk. J. A. M. A., 94:1552, 1930.

13. Alıneida Lima - Tumores medulares. Ed. Salvat, Madrid, 1943.

Calláo, $232\left(3 .^{\circ}\right.$ piso) - Buenos Aires, Argentina. 